\title{
Avaliação dos pacientes sobre os estressores em uma unidade coronariana*
}

\author{
Assessment of Stressors Among Inpatients of a Coronary Care Unit \\ Evaluación de los pacientes sobre factores generadores de estrés en una unidad \\ coronaria
}

\section{Carina Aparecida Marosti ${ }^{1}$, Rosana Aparecida Spadoti Dantas ${ }^{2}$}

\section{RESUMO}

Objetivo: identificar os fatores geradores de estresse para pacientes internados em uma Unidade Coronariana. Métodos: os dados foram coletados através de entrevista com 43 pacientes. Para a avaliação dos estressores foi utilizada uma escala tipo Likert de 4 pontos que avaliou a intensidade do estresse para 42 possíveis estressores, variando de 1 (não estressante) a 4 (muito estressante). Resultados: no geral, a média obtida para os itens foi 1,71 , caracterizando-os entre não estressantes e pouco estressantes na percepção dos pacientes. Os fatores mais estressantes foram: ter dor, não conseguir dormir, sentir falta do marido/esposa, ver a família por apenas alguns minutos e não poder mexer os braços. Conclusão: esses resultados podem auxiliar no planejamento da assistência de enfermagem.

Descritores: Estresse; Cuidados de enfermagem; Unidades de terapia intensiva; Doenças cardiovasculares

\begin{abstract}
Objective: This study aimed to identify stress-producing factors of hospitalized patients hospitalized in a coronary care unit. Methods: Data were collected through face-to-face interviews with 43 patients. The stressors were assessed with a four-point Likert scale, which evaluated stress intensity for 42 possible stressors, ranging from 1 (not stressful) to 4 (very stressful). Results: In general, the average score for the items was 1.71, characterizing them between not stressful and little stressful according to the patients' perception. The most stressful factors were: feeling pain, not being able to sleep, missing one's husband/wife, seeing one's family just a few minutes and not being able to move one's arms. Conclusion: These results can help in the planning of nursing care.
\end{abstract}

Keywords: Stress; Nursing care; Intensive care unit; Cardiovascular diseases

\section{RESUMEN}

Objetivo: identificar los factores generadores de estrés en pacientes internados en una Unidad Coronaria. Método: los datos fueron recolectados a través de una entrevista realizada a 43 pacientes. Para la evaluación de los estresores se utilizó una escala de tipo Likert de 4 pontos que evaluó la intensidad del estrés para 42 posibles estresores, variando de 1 (no estresante) a 4 (muy estresante). Resultados: en general, el promedio obtenido para los items fue de 1,71, siendo caracterizados como no estresantes y poco estresantes en la percepción de los pacientes. Los factores más estresantes fueron: tener dolor, no conseguir dormir, sentir falta del marido/esposa, ver a la familia por apenas algunos minutos y no poder mover los brazos. Conclusión: estos resultados pueden auxiliar en la planificación de la asistencia de enfermería. Descriptores: Estrés; Atención de enfermeria; Unidades de cuidados intensivos; Enfermedades cardiovasculares

\footnotetext{
* Trabalho extraído da Monografia de Conclusão do Curso de Especialização em Enfermagem Clínico-Cirúrgica, área de Cardiologia, da Escola de Enfermagem de Ribeirão Preto da Universidade de São Paulo. Ribeirão Preto (SP), Brasil.

${ }^{1}$ Enfermeira do Hospital das Clínicas de Ribeirão Preto. Ribeirão Preto (SP), Brasil.

${ }^{2}$ Professora Doutora do Departamento de Enfermagem Geral e Especializada da Escola de Enfermagem de Ribeirão Preto da Universidade de São Paulo. Ribeirão Preto (SP), Brasil.
} 


\section{INTRODUÇÃO}

O convívio com pacientes internados em uma Unidade Coronariana (UCO) suscitou-nos o interesse em aprofundar nosso conhecimento sobre quais são os fatores geradores de estresse para os doentes nessa unidade. Trata-se de uma área completamente equipada com todos os materiais necessários para detecção e tratamento das complicações das doenças cardíacas, e que traz, como conseqüência, estímulos que estão presentes no ambiente físico e social dessa unidade, os quais podem ser fontes de estresse para o paciente. Como exemplos de fatores geradores de estresse temos a perda da privacidade agravada pelo fato de homens e mulheres ficarem dispostos no mesmo ambiente, os desconfortos causados pela monitorização contínua, ausência de qualquer atividade de recreação para os pacientes e as sucessivas punções venosas ${ }^{(1)}$.

Estressores são definidos como sendo estímulos precedentes ou precipitantes de mudança e podem ser classificados em internos (aqueles que se originam dentro da pessoa como, por exemplo, uma febre) ou externos (aqueles que se originam fora da pessoa como, por exemplo, mudanças ambientais ou nas relações sociais) ${ }^{(2)}$. Estar submetido a um ambiente estressante pode gerar uma resposta fisiológica ao estresse que é influenciada pelo número de estressores, sua intensidade e duração. $\mathrm{O}$ estresse desencadeia uma série de respostas orgânicas e pode afetar processos que são relevantes para a hemostasia e trombogênese, levando ao aumento da ativação plaquetária e viscosidade do sangue, bem como a redução aguda do volume circulante plasmático. A patogênese de alterações induzidas pela exposição aguda ao estresse mental refere-se, principalmente, à isquemia miocárdica e à presença de arritmias ${ }^{(3)}$.

Os cuidados de enfermagem desenvolvidos pelos profissionais que atuam em UCO visam atender aos pacientes cardíacos detectando, inclusive, precocemente complicações decorrentes do seu estado clínico promovendo o restabelecimento hemodinâmico do doente e o favorecimento da sua recuperação. Entender como o paciente cardíaco se sente neste ambiente, pode auxiliar o enfermeiro a definir quais são os fatores estressantes nessas unidades, propiciando a construção de protocolos que transformem o setor em um local mais adequado e agradável ${ }^{(4)}$.

Devido à complexidade da UCO, podemos considerar que se trata de uma unidade de tratamento intensivo. Vários estudos têm investigado o estresse de pacientes internados nessas unidades, embora não abordem especificamente os pacientes $\operatorname{cardíacos}^{(4-5)}$. Assim, estabelecemos como finalidade do presente estudo identificar quais os fatores estressantes para os pacientes internados em uma UCO. A importância do estudo está fundamentada em todas as alterações fisiológicas, principalmente no sistema cardiovascular, as quais são desencadeadas pelo estresse. Acreditamos que, eliminar fontes de estresse pode contribuir para uma recuperação mais rápida do paciente internado em unidades coronarianas. Assim estabelecemos como objetivo do estudo avaliar os possíveis estressores de pacientes internados em uma Unidade Coronariana

\section{MÉTODOS}

O estudo é de caráter descritivo e exploratório, do tipo corte transversal, que foi desenvolvido na Unidade Coronariana de um hospital de ensino localizado no interior do Estado de São Paulo, após ter sido aprovado pelo Comitê de Ética do referido hospital. Uma amostra de conveniência foi formada por 43 pacientes que atenderem aos seguintes critérios de inclusão: ter acima de 21 anos; ter permanecido internado na UCO por um tempo mínimo de 48 horas, estar em condições clínicas que permitissem responder ao questionário e concordar em participar do estudo.

Os dados foram coletados por entrevistas individuais com os pacientes durante a internação na UCO, entre maio e setembro de 2004. Foi realizado um pré-teste com três pacientes internados na unidade, visando analisar a adequação do instrumento de coleta. Como não houve necessidade de mudanças no questionário, estes pacientes foram incluídos em nossa amostragem. Dentre os 43 sujeitos, $39(89,3 \%)$ solicitaram o auxílio da pesquisadora/enfermeira para anotarem as respostas, em decorrência de dificuldades físicas (como déficit de visão) ou cognitivas (não saber ler e/ou escrever).

$O$ instrumento de coleta continha dados para a caracterização sócio-demográfica e clínica dos participantes e a Escala de Estressores em Terapia Intensiva (EETI) ${ }^{(6-7)}$ em sua versão traduzida e validada para o português ${ }^{(5,8)}$. A EETI é composta por quarenta e duas perguntas e sua utilização visa identificar os fatores estressantes para o paciente internado em unidades de tratamento intensivo. Trata-se de uma escala do tipo Likert de quatro pontos em que (1) significa não estressante; (2) pouco estressante; (3) estressante; (4) muito estressante. A avaliação dos resultados é obtida pela soma dos valores atribuídos a cada um dos 42 itens, variando de 42 a 168, sendo que quanto maior o valor, maior o estresse percebido pelo paciente.

Os dados foram processados e analisados no programa Statistical Package for Social Science (SPSS) versão 10.0. Para a análise descritiva das variáveis foram utilizadas medidas de posição (média, mediana) e variabilidade (desvio-padrão) para as variáveis contínuas (idade, tempo de internação, escala de estressores) e de freqüência simples para as variáveis categóricas. Para 
verificar a confiabilidade da escala de estressores através de sua consistência interna foi utilizado o Coeficiente Alfa de Cronbach.

\section{RESULTADOS}

A amostra deste estudo constou de 43 pacientes internados na Unidade Coronariana do referido hospital. A caracterização sócio-demográfica dos participantes está apresentada na Tabela 1. A idade média dos pacientes foi de 56 anos (S.D. $=11,9$ ), variando de 21 a 78 anos. A maioria era do sexo masculino (31 pacientes; $72,1 \%$ do total), sendo $24(55,8 \%)$ casados e $8(18,6 \%)$ viúvos. Quanto à situação profissional, apenas $14(32,6 \%)$ desempenhavam atividades remuneradas na época da internação. Em relação à escolaridade, apenas 7 pacientes $(16,3 \%)$ tinham mais que oito anos de estudo formal, o que pode ter contribuído para o pequeno número de pacientes que preencheram sozinhos os instrumentos.

Quanto à caracterização clínica dos participantes (Tabela 2), no que se refere ao diagnóstico na internação, o mais freqüente foi infarto do miocárdio $(53,5 \%)$, seguido de angina $(25,6 \%)$, insuficiência cardíaca congestiva descompensada $(7,0 \%)$, edema agudo de pulmão $(4,7 \%)$ e outros $(9,3 \%)$. Por considerarmos que o uso de psicotrópicos poderia afetar a percepção do paciente com relação ao estresse da internação na UCO, avaliamos o uso destes medicamentos na prescrição médica, no dia da entrevista. Constatamos que 31 pacientes $(72,1 \%)$ não haviam recebido qualquer droga psicotrópica nas 24 horas que antecederam a coleta dos dados. A maioria dos pacientes estava vivenciando a internação em uma unidade de terapia intensiva pela primeira vez $(76,7 \%)$, já estavam internados na unidade, em média, há mais de 48 horas e utilizavam, no mínimo, 2 equipamentos para o seu controle clínico.

No que se refere ao instrumento escolhido para avaliar os estressores nos pacientes internados na UCO, optamos por utilizar um instrumento já validado para o português ${ }^{(5)}$, o qual mostrou-se válido e confiável em outros estudos ${ }^{(8-9)}$. O alfa de Cronbach, medida da confiabilidade do instrumento, foi de 0,93 o que reflete a alta consistência interna dos itens presentes na escala.

O intervalo possível para a escala era de 42 (42 questões x 1) até 168 (42 x 4), sendo que, quanto maior o valor da somatória dos itens, maior era o estresse percebido na UCO. $\mathrm{Na}$ amostra estudada obtivemos um valor médio de 72,11 (S.D. = 21,7), com intervalo de 43 a 134. A posição das pontuações dadas aos itens da escala, pelos pacientes, nos permitiu levantar quais os itens considerados como maiores fontes geradoras de estresse, e estão apresentados na Tabela 3.

Podemos avaliar os resultados desta escala também analisando a média das respostas aos itens, ou seja, a soma dos pontos obtidos em todos os itens, dividida por 42 (total de itens da escala), e em nosso estudo obteve o valor de 1,7 (S.D. $=0,5)$, com intervalo das médias entre 1 e 3,2 . Ao considerar que os valores de estresse na escala são: 1 (não estressante), 2 (pouco estressante), 3 (estressante) e 4 (muito estressante), no geral, os pacientes estudados consideraram a internação na UCO como sendo uma experiência entre não estressante e pouco estressante.

Os resultados obtidos indicam que os fatores mais estressantes, para os pacientes, foram: ter dor, não

Tabela 1: Caracterização sócio-demográfica dos participantes

\begin{tabular}{|c|c|c|c|c|}
\hline Variável $(\mathrm{N}=43)$ & Intervalo & Mediana & Média (DP) & $\mathrm{N}(\%)$ \\
\hline Idade (anos) & $21-78$ & 58,2 & $56,73(11,9)$ & \\
\hline Escolaridade (anos) & $0-18$ & 4,0 & $5,0(4,1)$ & \\
\hline Até 4 anos & & & & $27(62,8)$ \\
\hline De 5 a 8 anos & & & & $9(20,9)$ \\
\hline De 9 a 11 anos & & & & $4(9,3)$ \\
\hline Acima de 12 anos & & & & $3(7,0)$ \\
\hline \multicolumn{5}{|l|}{ Sexo } \\
\hline Feminino & & & & $12(27,9)$ \\
\hline Masculino & & & & $31(72,1)$ \\
\hline \multicolumn{5}{|l|}{ Estado Civil } \\
\hline Casado & & & & $24(55,8)$ \\
\hline Solteiro & & & & $6(14,0)$ \\
\hline Viúvo & & & & $8(18,6)$ \\
\hline Desquitado/divorciado & & & & $5(11,6)$ \\
\hline \multicolumn{5}{|l|}{ Situação Profissional } \\
\hline Ativo & & & & $14(32,6)$ \\
\hline Aposentado & & & & $18(41,9)$ \\
\hline Aposentado com atividade remunerada & & & & $3(7,0)$ \\
\hline Do lar & & & & $2(4,7)$ \\
\hline Desempregado & & & & $2(4,7)$ \\
\hline Afastado por problema de saúde & & & & $4(9,3)$ \\
\hline
\end{tabular}


Tabela 2: Caracterização clínica dos participantes

\begin{tabular}{|c|c|c|c|c|}
\hline Variável & Intervalo & Mediana & $\begin{array}{l}\text { Média } \\
\text { (D.P.) }\end{array}$ & $\mathrm{N}(\%)$ \\
\hline \multicolumn{5}{|l|}{ Diagnóstico na internação } \\
\hline IAM & & & & 2353,5 \\
\hline Angina & & & & 1125,6 \\
\hline ICC & & & & 37,0 \\
\hline EAP & & & & 24,7 \\
\hline Outros & & & & 49,3 \\
\hline \multicolumn{5}{|l|}{ Uso de psicotrópicos } \\
\hline Sim & & & & 1227,9 \\
\hline Não & & & & 3172,1 \\
\hline \multicolumn{5}{|l|}{ Primeira internação } \\
\hline Sim & & & & 3376,7 \\
\hline Não & & & & 1023,3 \\
\hline Tempo de internação & $2-11$ & 3,0 & $2,31,9$ & \\
\hline Até 2 dias & & & & 1944,2 \\
\hline De 3 a 5 dias & & & & 1841,9 \\
\hline Acima de 6 dias & & & & 614,0 \\
\hline Número de equipamentos & $2-5$ & 2,0 & $2,30,7$ & \\
\hline
\end{tabular}

conseguir dormir; sentir falta do marido ou esposa, ver a família e amigos por poucos minutos e não conseguir mexer as mãos e braços devido às vias intravenosas. Alguns itens foram classificados como menos estressantes, na visão dos pacientes internados na UCO: ser examinado por médicos e enfermeiros a todo o momento, ter a equipe de enfermagem realizando tarefas ao redor do leito a todo momento e escutar o telefone tocar.

\section{DISCUSSÃO}

Entre os sujeitos do estudo, Ter dor foi classificado como o principal estressor, resultado também

Tabela 3: Posição dos estressores na UCO, segundo a amostra estudada

\begin{tabular}{|c|c|c|c|}
\hline ESTRESSORES & POSIÇÃO & MÉDIA & D. P. \\
\hline Ter dor & 1 & 3,21 & 1,10 \\
\hline Não conseguir dormir & 2 & 2,51 & 1,40 \\
\hline Sentir falta do marido ou esposa & 3 & 2,44 & 1,24 \\
\hline Ver a família e amigos por poucos minutos & 3 & 2,44 & 1,16 \\
\hline Não conseguir mexer mãos e braços devido vias intravenosas & 4 & 2,28 & 1,18 \\
\hline Não ter controle de si mesmo & 5 & 2,26 & 1,25 \\
\hline Ser furado por agulhas & 6 & 2,19 & 1,18 \\
\hline Ter que ficar olhando para o teto & 7 & 2,16 & 1,27 \\
\hline Não saber quando as coisas serão feitas & 8 & 2,07 & 1,16 \\
\hline Escutar o alarme do monitor cardíaco disparar & 9 & 2,02 & 1,18 \\
\hline Escutar o gemido de outros pacientes & 10 & 1,98 & 1,18 \\
\hline Ter luzes acesas constantemente & 11 & 1,95 & 1,54 \\
\hline Ter a equipe falando termos desconhecidos & 11 & 1,95 & 1,06 \\
\hline Escutar o barulho e alarme dos equipamentos & 12 & 1,93 & 1,10 \\
\hline Sons e ruídos desconhecidos & 13 & 1,79 & 1,03 \\
\hline Não saber que horas são & 14 & 1,74 & 1,07 \\
\hline Ter que usar oxigênio & 15 & 1,72 & 1,12 \\
\hline Não ter privacidade & 15 & 1,72 & 1,00 \\
\hline Ter tubos no nariz/boca & 16 & 1,70 & 1,12 \\
\hline Não ter explicações sobre o tratamento & 17 & 1,63 & 1,04 \\
\hline Estar amarrado por tubos & 18 & 1,58 & 1,09 \\
\hline Cama e/ou travesseiro não confortáveis & 18 & 1,58 & 0,87 \\
\hline Ter máquinas estranhas ao redor & 19 & 1,53 & 0,98 \\
\hline Medir a pressão arterial muitas vezes ao dia & 20 & 1,51 & 0,91 \\
\hline Ver as bolsas de soro penduradas sobre a cabeça & 20 & 1,51 & 0,91 \\
\hline Sentir cheiros estranhos & 21 & 1,49 & 0,91 \\
\hline Enfermagem e médico falando muito alto & 22 & 1,47 & 0,82 \\
\hline Ter homens e mulheres internados no mesmo ambiente & 22 & 1,47 & 0,88 \\
\hline Não saber que dia é hoje & 22 & 1,47 & 0,96 \\
\hline Estar em um ambiente muito quente ou muito frio & 22 & 1,47 & 0,93 \\
\hline Ser incomodado & 23 & 1,44 & 0,76 \\
\hline Assistir aos cuidados médicos e de enfermagem realizados em outros pacientes & 24 & 1,42 & 0,90 \\
\hline Sentir que a equipe de enfermagem está mais atenta aos equipamentos do que a você & 25 & 1,40 & 0,90 \\
\hline Ter sede & 26 & 1,37 & 0,87 \\
\hline Não saber onde está & 27 & 1,33 & 0,77 \\
\hline Ser cuidado por médicos desconhecidos & 28 & 1,28 & 0,66 \\
\hline A enfermeira não se apresentar pelo nome & 29 & 1,26 & 0,65 \\
\hline Sentir que o enfermeiro está muito ocupado & 29 & 1,26 & 0,69 \\
\hline Ser acordado pela equipe de enfermagem & 29 & 1,26 & 0,58 \\
\hline Ser examinado por médicos e enfermeiros a todo o momento & 30 & 1,21 & 0,55 \\
\hline Ter a enfermagem realizando tarefas ao redor do leito a todo o momento & 31 & 1,09 & 0,36 \\
\hline Escutar o telefone tocar & 32 & 1,05 & 0,21 \\
\hline
\end{tabular}


encontrado por outros pesquisadores ${ }^{(4-5,8-9)}$, tanto na visão dos pacientes e dos seus familiares, quanto na avaliação da equipe de enfermagem.

A presença da dor é algo tão desconfortável que ultrapassa outras inconveniências experimentadas nas unidades de tratamento intensivo ${ }^{(5)}$. A dor pode ser decorrente da doença cardíaca, do estado psicológico, dos procedimentos diagnósticos e terapêuticos e do próprio ambiente da unidade de terapia intensiva ${ }^{(10)}$. Somente esse item foi avaliado pelos pacientes internados na UCO como sendo estressante ou muito estressante. Os itens que ficaram classificados, segundo as médias das respostas, entre $2^{\circ}$ e $9^{\circ}$ lugares foram considerados como pouco estressantes ou estressantes.

$\mathrm{O}$ segundo item, avaliado entre pouco estressante e estressante, foi não conseguir dormir, resultado encontrado em outros estudos ${ }^{(5,11)}$. O ambiente da UCO, a presença da dor, o medo do desconhecido, a presença de equipamentos sonoros e luminosos e muitos outros fatores combinam-se para dificultar o sono/repouso dos pacientes. O sono pode ser definido como um estado complexo de aparente repouso normal e periódico, qualitativa e quantitativamente variável, caracterizado pela suspensão parcial da percepção dos fatores ambientais e da motricidade voluntária. Constitui-se em um estado peculiar de consciência, diverso do coma e da anestesia, por ser funcional e periódico, isto é, espontaneamente reversível e recorrente ${ }^{(12)}$. O período do sono é muito importante, pois várias transformações metabólicas e fisiológicas ocorrem neste período e interferem no desempenho adequado das funções orgânicas ${ }^{(12)}$.

Sentirfalta do marido / esposa e ver a família por poncos minutos foram dois estressores que ocuparam o terceiro lugar. Esse resultado não condiz com a literatura encontrada, na qual esses estressores aparecem com menor grau de importância ${ }^{(5,11,13)}$. Podemos justificar essa diferença no tipo de política adotada pelas instituições estudadas, no que se refere ao horário e presença de familiares em unidades de terapia intensiva. Vale ressaltar, que no hospital onde foi realizado nosso estudo, o horário de visita é fixo, e apenas um número limitado de visitantes pode adentrar a unidade para ver o paciente.

O quarto estressor foi não conseguir mexer os braços e mãos devido às vias intravenosas. Esse item também foi encontrado na literatura como um dos mais estressantes, ocupando o sétimo lugar no ranking total ${ }^{(5)}$.

O sentimento de não ter controle de si mesmo foi o estressor que apareceu em quinto lugar, o que corrobora com a literatura encontrada ${ }^{(5,13)}$. Durante a internação em unidades intensivas, o paciente regressa para comportamentos infantis, a um estado de dependência, tendo que aceitar que estranhos fiquem responsáveis pela sua higiene, alimentação ou até mesmo trocar a posição no leito. A perda da independência pode gerar angústia e a sensação de impotência, a principal causa de instabilidade psicológica nesse contexto ${ }^{(14)}$.

Ter tubos no nariz e na boca assim como estar amarrado por tubos foram os principais estressores encontrados na literatura ${ }^{(5,7,13)}$. No entanto, no presente estudo, esses itens não aparecem no ranking dos maiores estressores, ocupando os $16^{\circ}$ e $18^{\circ}$ lugares, respectivamente. Cabe ressaltar que por ter sido realizado em uma UCO e não em uma UTI, propriamente dita, nenhum dos pacientes entrevistados havia tido a necessidade de ser entubado ou de usar sondas gástricas. Apenas dois pacientes (4,7\%) utilizaram o balão intra-aórtico e sete pacientes $(16,3 \%)$ permaneceram com introdutor na artéria femoral após angioplastia coronariana transluminal percutânea (ACTP) ou com monitorização invasiva da pressão arterial.

Foi encontrado que a maioria dos pacientes considerou a falta de privacidade como sendo pouco estressante, resultado que condiz com a literatura consultada ${ }^{(5)}$. Os pacientes enfrentam a internação na UTI como sendo um dos recursos para restaurar sua saúde. Nesta perspectiva, a privacidade pode não ser considerada como prioridade para eles ${ }^{(15)}$.

Acreditamos que, para os profissionais da enfermagem, é extremamente importante conhecer os reais fatores que causam estresse ao paciente internado na UCO, visto que trabalhos anteriores ${ }^{(7,16)}$ mostram que a avaliação da equipe de enfermagem nem sempre condiz com a avaliação do paciente. Uma avaliação distorcida impede a equipe de discriminar adequadamente os fatores que causam estresse para o paciente e a equipe de saúde pode estar projetando seus sentimentos nesse indivíduo ${ }^{(17)}$.

A atuação específica desses profissionais sobre os fatores estressantes para o paciente pode ser a chave para a humanização da UTI, favorecendo também a melhor evolução do paciente ${ }^{(15,18)}$.

\section{CONCLUSÕES}

$\mathrm{Na}$ amostra estudada, a internação na UCO foi avaliada, no geral, como sendo pouco estressante (média dos itens $=1,71$ ). Os maiores estressores levantados nesse estudo condizem com a literatura (exceto ver a família por poucos minutos e sentir falta do marido/esposa) e foram: ter dor, não conseguir dormir, não conseguir mexer os braços/mãos e não ter controle de si mesmo.

Diante do exposto, faz-se necessário replanejar a assistência de enfermagem ao paciente internado nesta unidade, enfocando a dor como um sinal importante para avaliar seu bem-estar físico e psicológico. A dor deve ser controlada e mensurada na UCO, com o mesmo rigor que são avaliados os sinais vitais dos pacientes, uma vez que a dor pode provocar o agravamento do quadro clínico do doente cardíaco. Além disso, deve haver uma interação com a equipe médica, visto que são eles que 
prescrevem os analgésicos. Em relação ao fato de não conseguir dormir, a equipe de enfermagem pode tomar algumas medidas como: evitar colocar horário de medicação durante a noite, falar baixo, evitar abrir e fechar a porta da UCO diversas vezes, diminuir ao máximo as luzes acesas, adotar medidas de conforto, tanto para a unidade do paciente como para o ambiente, assim como evitar aglomerado de pessoas durante a noite. Essas recomendações devem ser adotadas também por toda equipe multidisciplinar.

É importante lembrar que a falta do parceiro, assim como o período curto de visita são fatores muito estressantes para o paciente. Na medida do possível, as enfermeiras de plantão devem avaliar a situação de cada paciente e do plantão em si, permitindo visitas fora do horário pré-determinado, ou mesmo permitindo a maior permanência das visitas na UCO, assim como o revezamento dos visitantes. Em relação a não ter controle de si mesmo, faz-se necessário a avaliação multidisciplinar da necessidade de acompanhamento psicológico. Também maiores informações a respeito do processo de sua recuperação devem ser fornecidas ao paciente pela equipe. Concordamos com outros autores, quando referem que os pacientes devem participar ativamente do processo de seu tratamento e, mais que isso, que eles devem se sentir envolvidos nesse processo. A internação na UCO é uma difícil experiência na vida dos pacientes e de seus familiares. Assim, o controle do estresse permitirá melhor condição física e psíquica do paciente na sua recuperação, além de promover um ambiente mais humanizado nessas unidades.

\section{REFERÊNCIAS}

1. Gomes AM. Enfermagem na unidade de terapia intensiva. 2a.ed. São Paulo: EPU; 1988.

2. Potter PA, Perry AG. Fundamentos de enfermagem: conceitos, processo e pratica. 4a.ed. Rio de Janeiro: Guanabara Koogan; 1999.

3. Malyszko J, Urano T, Takada Y, Takada A. Stress-dependent changes in fibrinolysis, serotonin and platelet aggregation in rats. Life Sci. 1994; 54(17):1275-80.
4. Seidler H, Moritz RD. Recordações dos principais fatores que causam desconforto nos pacientes durante a sua internação em Unidade de Terapia Intensiva. Rev Bras Ter Intensiva. 1998; 10(3):112-7.

5. Novaes MA, Aronovich A, Ferraz MB, Knobel E. Stressors in ICU: patients' evaluation. Intensive Care Med. 1997; 23(12):1282-5.

6. Ballard KS. Identification of environmental stressors for patients in a surgical intensive care unit. Issues Ment Health Nurs. 1981; 3(1-2):89-108.

7. Cochran J, Ganong LH. A comparison of nurses' and patients' perceptions of intensive care unit stressors. J Adv Nurs. 1989; 14(12):1038-43.

8. Novaes MA, Knobel E, Bork AM, Pavão OF, NogueiraMartins LA, Ferraz MB. Stressors in ICU: perception of the patient relatives and health care team. Intensive Care Med. 1999; 25(12):1421-6.

9. Gois CFL, Dantas RAS. Estressores em uma unidade pósoperatória de cirurgia torácica: avaliação da enfermagem. Rev Latinoam Enfermagem. 2004; 12(1):22-7.

10. Ferguson JA. Pain following coronary artery bypass grafting: an exploration of contributing factors. Intensive Crit Care Nurs. 1992; 8(3):153-62.

11. Nastasy EL. Identifying environmental stressors for cardiac surgery patients in a SICU. In: Proceedings of the 12th Annual National Teaching Institute of AACN. New Port Beach, Califórnia: AACN; 1985. p. 357.

12. Porto CC. Semiologia médica. $4 a$ ed. Rio de Janeiro: Guanabara Koogan; 2001.

13. Soehren P. Stressors perceived by cardiac surgical patients in the intensive care unit. Am J Crit Care. 1995; 4(1):71-6.

14. Carr JA, Powers MJ. Stressors associated with coronary bypass surgery. Nurs Res. 1986; 35(4):243-6.

15. Elpern EH, Patterson PA, Gloskey D, Bone RC. Patients' preferences for intensive care. Crit Care Med. 1992; 20(1):437.

16. Cornock MA. Stress and the intensive care patient: perceptions of patients and nurses. J Adv Nurs. 1998; 27(3):518-27.

17. Ross SE, MacKay RC. Postoperative stress. Do nurses accurately their patients? J Psychosoc Nurs Ment Health Serv. 1986; 24(4):16-22.

18. Yarcheski A, Knapp-Spooner C. Stressors associated with coronary bypass surgery. Clin Nurs Res. 1994; 3(1):57-68. 